\title{
11. $\ddot{\boldsymbol{U} b e r}$ \\ die sogenannten Kern- und Konvergenzpunkte \\ der ,kristallinisch-flüssigen Phase"6 \\ von Paraazoxyphenetol; \\ von Georg Wulff.
}

Erwärmt man am Heizmikroskrop eine zwischen Objekt. und Deckgläschen eingeschlossene Schicht von Paraazoxyphenetol bis zum isotropen Zustande, und kühlt sie langsam ab, so sieht man die Tropfen sich bilden, die in der Mitte ein kreisrundes dunkles Feld besitzen, dessen Mittelpunkt noch dunkler ist. Diese Felder wurden von O. Leh. mann "Kernpunkte" genannt. Durch ZusammenflieBen von Tropfen bildet sich eine kontinuierliche Masse, in der außer den Kernpunkten noch andere ähuliche dunkle Gebilde sich feststellen lassen, die ebenfalls ein dunkles Feld besitzen, dessen Form aber nicht kreisrund, sondern viereckig, meist quadratisch ist. Diese Gebilde wurden von 0 . Lehmann „Konvergenzpunkte" genannt.

Die Kern- und Konvergenzpunkte zeigen folgende Eigenschaften.

1. Sio sind Verdichtungen in der flüssigen Masse, da sie der Bewegung der kleinen, durch Strömungen mitgerissenen Körperchen einen unüberwindlichen Widerstand leisten.

2. Das durch die Kern- und Konvergenzpunkte durchgegangene Licht erweist sich polarisiert. Wenn man den Analysator (oder Polarisator) einschaltet, so merkt man innerhalb der runden und quadratischen Felder der Kern- und Konvergenzpunkte einen diametralen dunklen Balken. Dreht man den Analysator, so drehen sich auch die Balken, doch geht diese Drehung in den Kernpunkten im Sinne der Drehung des Nicols ror, in den Konvergenzpunkten aber ist sie entgegengesetzt. 
3. Die Kern- und Konvergenzpunkte sind kettenartig geordnet, wobei immer zwischen zwei Kernpunkten ein Konvergenzpunkt eingeschaltet wird.

4. Die Kern- und Konvergenzpunkte ziehen sich gegenseitig an.

Wir werden alle diese Eigenschaften durch die Annahme erklären, daB die sogenannte trübe Phase von Paraazoxy: phenetol eine kolloidale Lösung ist, deren Suspension an der Oberfläche der Flüssigkeit ein kapillares Häutchen bildet und zu Koagulationsgebilden von eigenartiger Struktur sich zusammenzuziehen fähig ist, die mit dem Häutchen in Verbindung stehen. Diese Koagulationen rufen im Häutchen und in der Flüssigkeit Spannungen hervor und werden selbst unter der Wirkung der Spannungen gebildet.

Nach dieser Annahme wird die Oberfläche eines Tropfens mit einem Kernpunkte den Spannungen ausgesetzt, die durch die Anwesenheit des Kernpunktes bedingt sind. Die Spannungen sollen deshalb meridional durch den Kernpunkt verlaufen. Der Kernpunkt soll eine konzentrische Struktur besitzen und die Polarisationsebenen sollen tangential zu den konzentrischen Schalen verlaufen. Damit wird das Verhalten der Kernpunkte zu dem polarisierten Lichte vollständig erklärt.

Was die Konvergenzpunkte anbetrifft, so beachte man, daB sie sich beim ZusammenflieBen von Tropfen bilden. Dabei wird der Berührungspunkt $O$ der Tropfen (Fig. 1) den Spannungen ausgesetzt, die in der unmittelbaren Nähe von $O$ als ein System rechtwinkeliger Hyperbeln angesehen werden kann. Die unter solchen Spannungen sich bildende Koagulation muB eine hyperbolische Struktur annehmen, und zwar müssen die Polarisationsebenen in einer solchen Koagulation tangential zu einem System von ebenfalls rechtwinkeligen Hyperbeln verlaufen, die orthogonal zu dem ersteren System steht. Man kann leicht beweisen, daB eine solche Anordnung der

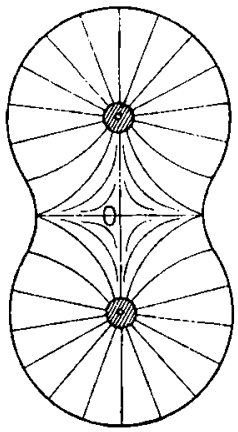

Fig. 1. Polarisationsebenen eine Drehung des zentralen Balkens zur Folge haben soll, die der Drehung des Analysators entgegengesetzt ist. 
184 G. Wulff. Die sogenannten Kern- u. Konvergenzpunkte usu.

Es sei

$$
y x=m
$$

die Gleichung einer von der Hyberbeln und

$$
y=a x
$$

die Gleichung der Geraden, die die Richtung der Polarisationsebenen des Analysators angibt. Der Punkt $M$ der Hyperbel (Fig. 2), in dem die Tangente senkrecht zu $O A$ ist, erscheint

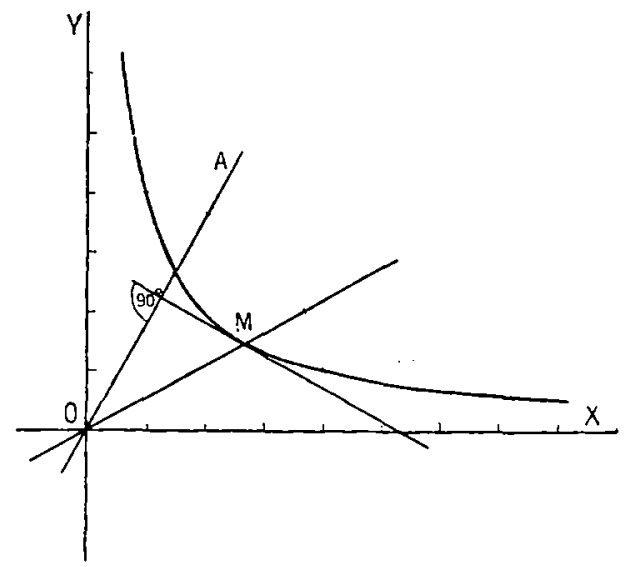

Fig. 2.

dunkel. Es ist aber leicht $z u$ beweisen, $d_{a} B$ die Gleichung der Geraden $O M$

$$
y=\frac{1}{a} x
$$

ist, also unabhängig von $m$. Der Ort dunkler Punkte für das System ist eine Gerade, die als ein gerader dunkler Balken erscheint. Die Neigung des Balkens zu $O x$ ist gleich der Neigung der Polarisationsebene $O A$ des Analysators zu $O y$, es drehen sich also $O A$ und $O M$ in entgegengesetzter Richtung.

Wenn mehrere Tropfen zusammentließen, wird zwischen je zwei Kernpunkten ein Konvergenzpunkt eingeschaltet, und die Anordnung nach Ketten wird selbstrerständlich, ebenso wie ihre durch die Spannungen bedingte gegenseitige Anziehung. Moskau, Städtische Universität, Kristallogr. Laboratorium.

(Eingegangen 7. April 1911.) 